\title{
Segetal Weed Vegetation Database of Greece
}

\author{
Erwin Bergmeier
}

\begin{abstract}
The Segetal Weed Vegetation Database of Greece (GIVD ID EU-GR-004) attempts to document the vanishing plants and vegetation of segetal fields used in a traditional manner without herbicide application. At present it includes chiefly unpublished records of southern mainland Greece and recently published data from the island of Crete. Its focus is on the documentation of rare weed species occurrences and on the option of monitoring plants, vegetation and land use.
\end{abstract}

Keywords: Aegean; Mediterranean vegetation; traditional agriculture.

GIVD Database ID: EU-GR-004

Last update: 2011-07-06

\section{Segetal Weed Vegetation Database of Greece}

Scope: Plot samples of arable fields and their margins as well as of recent fallows in Greece and the Aegean. It is intended to widen the geographical scope to Turkey and the southern Balkans. Special attention is paid to segetal fields (wheat, oats, barley, rye) of traditional agriculture without herbicide application.

Status: ongoing capture Period: 1983-2010

Database manager(s): Erwin Bergmeier (erwin.bergmeier@bio.uni-goettingen.de)

Owner: Erwin Bergmeier (private)

Web address: [NA]

Availability: according to a specific agreement

Database format(s): TURBOVEG

Online upload: no

Online search: no

Publication: [NA]

Plot type(s): normal plots

Export format(s): TURBOVEG, Excel

Non-overlapping plots: 200

Plot-size range: $16-50 \mathrm{~m}^{2}$

Total plot observations: 200

Estimate of existing plots: 250

Completeness: $80 \%$

Countries: GR: $100.0 \%$

Forest: [NA] — Non-forest: [NA]

Guilds: all vascular plants: $100 \%$

Environmental data: altitude: $100 \%$; slope aspect: $100 \%$; slope inclination: $100 \%$; soil depth: $100 \%$; other soil attributes: $100 \%$

Performance measure(s): cover: $100 \%$

Geographic localisation: GPS coordinates (precision $25 \mathrm{~m}$ or less): $40 \%$; point coordinates less precise than GPS, up to $1 \mathrm{~km}$ : $55 \%$; small grid (not coarser than $10 \mathrm{~km}$ ): $5 \%$

Sampling periods: $1980-1989: 1.0 \%$; 1990-1999: 60.0\%; 2000-2009: 35.0\%; 2010-2019: 4.0\%

Information as of 2012-07-12; further details and future updates available from http://www.givd.info/ID/EU-GR-004

Erwin Bergmeier (erwin.bergmeier@bio.uni-goettingen.de)

Vegetation Analysis \& Phytodiversity, University of Göttingen, Untere Karspüle 2, 37073 Göttingen, GERMANY 